\title{
Existence and stability results for neutral stochastic delay differential equations driven by a fractional Brownian motion
}

\author{
R. Maheswari ${ }^{1 *}$, S. Karunanithi ${ }^{2}$ \\ ${ }^{1}$ Department of Mathematics, Sri Eshwar College of Engg., Coimbatore-641 202, Tamilnadu, \\ ${ }^{2}$ Department of Mathematics, Kongunadu Arts and Science College (Autonomous), Coimbatore-641 029, Tamilnadu, India \\ *Corresponding author E-mail:mahesenthil12@gmail.com
}

Copyright $\odot$ 2015R. Maheswari, S. Karunanithi. This is an open access article distributed under the Creative Commons Attribution License, which permits unrestricted use, distribution, and reproduction in any medium, provided the original work is properly cited.

\begin{abstract}
In this paper we investigate the existence, uniqueness, asymptotic behavior of mild solutions to neutral stochastic differential equations with delays driven by a fractional Brownian motion in a Hilbert space. The cases of finite and infinite delays are analyzed.
\end{abstract}

Keywords: Asymptotic Behaviors; Delays; Fractional Brownian Motion; Mild Solution; Wiener Integral.

\section{Introduction}

The theory of stochastic differential equations driven by a fractional Brownian motion ( $\mathrm{fBm}$ ) has been studied intensively in recent years [1], [2], [3], [4], and [5]. The fBm received much attention because of its huge range of potential applications in several fields like telecommunications, networks, finance markets, biology and so on [6], [7], [8], [9]. Moreover, one of the simplest stochastic processes that is Gaussian, self-similar, and has stationary increments is $\mathrm{fBm}$ [10]. In particular, $\mathrm{fBm}$ is a generalization of the classical Brownian motion, which depends on a parameter $\mathrm{H} \in$ $(0,1)$ called the Hurst index [9]. It should be mentioned that when $\mathrm{H}=\frac{1}{2}$, the stochastic process is a standard Brownian motion; when $\mathrm{H} \neq \frac{1}{2}$, it behaves completely in a different way than the standard Brownian motion, in particular neither is a semimartingale nor a Markov process. It is a self-similar process with stationary increments and has a long-memory whenH $\neq \frac{1}{2}$. These significant properties make $\mathrm{fBm}$ a natural candidate as a model for noise in a wide variety of physical phenomena such as mathematical finance, communication networks, hydrology and medicine. The existence and uniqueness of mild solutions for a class of stochastic differential equations in a Hilbert space with a standard, cylindrical fBm with the Hurst parameter in the interval $\left(\frac{1}{2}, 1\right)$ has been studied [11]. Maslowski and Nualart [12] have studied the existence and uniqueness of a mild solution for nonlinear stochastic evolution equations in a Hilbert space driven by a cylindrical $\mathrm{fBm}$ under some regularity and boundedness conditions on the coefficients. Recently, Caraballo and et al [13] investigated the existence and uniqueness of mild solutions to stochastic delay equations driven by $\mathrm{fBm}$ with Hurst parameter $H \in\left(\frac{1}{2}, 1\right)$. An existence and uniqueness result of mild solutions for a class of neutral stochastic differential equation with finite delay, driven by an $\mathrm{fBm}$ in a Hilbert space has been investigated [14] in Boufoussi and Hajji. The asymptotic behavior of solutions for stochastic differential equations with $\mathrm{fBm}$ has only been investigated by a few authors [13], [14], [15]. Moreover Nguyen [16] has studied the asymptotic behaviors of mild solutions to neutral stochastic differential equations driven by an $\mathrm{fBm}$. Motivated by this consideration in this paper we investigate the existence and uniqueness and asymptotic behaviors of mild solutions for a neutral stochastic differential equation with finite or infinite delays driven by $\mathrm{fBm}$ in the following form 


$$
\begin{aligned}
& d[x(t)-g(t, x(t-r(t)))]= {[A x(t)+f(t, x(t-\rho(t)))] d t+h(t, x(t-\delta(t))) d W(t) } \\
&+\sigma(t) d B_{Q}^{H}(t) ; t \geq 0 \\
& x(t)=\phi(t), t \in(-\tau, 0](0<\tau \leq \infty)
\end{aligned}
$$

where $\mathrm{A}$ is an infinitesimal generator of an analytic semigroup of bounded linear operators, $(\mathrm{S}(\mathrm{t}))_{\mathrm{t} \geq 0}$ in a Hilbert space $X$ with norm $\|\cdot\|, B_{Q}^{H}(t)$ denotes an $\mathrm{fBm}$ with $\mathrm{H}>\frac{1}{2}$ on a real and separable Hilbert space $\mathrm{Y}, \mathrm{r}, \rho, \delta:[0, \infty) \rightarrow[0, \tau)$ are continuous, $f, g:[0, \infty) \times X \rightarrow X, h:[0, \infty) \times X \rightarrow \mathbb{L}_{2}^{0}, \sigma:[0, \infty) \rightarrow \mathcal{L}_{Q}^{0}(Y, X)$, the initial data $\phi \in C((-\tau, 0], X)$ the space of all continuous functions from $(-\tau, 0]$ to $\mathrm{X}$ and has finite second moments. Further we assume $\mathrm{W}$ and $\mathrm{B}^{\mathrm{H}}$ are independent. The main tool of this paper is the fixed point theory which was proposed by Burton [17].

\section{Preliminaries}

In this section we first recall the $\mathrm{fBm}$ as well as the Wiener integral with respect to it. We also establish some important results which will be needed throughout the paper. Let $(\Omega, \mathcal{F}, \mathrm{P})$ be a complete probability space and $\mathrm{T}>0$ be an arbitrary fixed horizon. Let $\mathrm{X}$ and $\mathrm{Y}$ be two real, separable Hilbert spaces and $\mathrm{L}(\mathrm{Y}, \mathrm{X})$ be the space of bounded linear operators from $\mathrm{Y}$ to $\mathrm{X}$.

Let $\mathbb{L}_{2}^{0}(\mathrm{Y}, \mathrm{X})$ be separable Hilbert space with respect to the Hilbert-Schmidt norm $\|\cdot\|_{L_{2}^{0}}$. Let $\mathcal{L}_{\mathrm{Q}}^{0}(\mathrm{Y}, \mathrm{X})$ be the space of all $\psi \in \mathcal{L}(\mathrm{Y}, \mathrm{X})$ such that $\psi \mathrm{Q}^{\frac{1}{2}}$ is a Hilbert Schmidt operator. The norm is given be $\|\psi\|_{\mathcal{L}_{\mathrm{Q}}^{0}}^{2}=\left\|\psi \mathrm{Q}^{\frac{1}{2}}\right\|=\operatorname{tr}\left(\psi Q \psi^{*}\right)$. Then $\psi$ is called a $Q$ Hilbert - Schmidt operator from $\mathrm{Y}$ to X.Let $\mathcal{L}_{2}^{0}(\Omega, X)$ denote the space of $\mathcal{F}_{0^{-}}$measurable, $\mathrm{X}$-valued and square integrable Stochastic processes. Consider a time interval $[0, \mathrm{~T}]$ with arbitrary fixed horizon $\mathrm{T}$ and let $\left\{\beta^{\mathrm{H}}(\mathrm{t}), \mathrm{t} \in[0, \mathrm{~T}]\right\}$ be a two sided one dimensional $\mathrm{fBm}$ with Hurst parameter $\mathrm{H} \in\left(\frac{1}{2}, 1\right)$ with the covariance function

$\mathrm{R}_{\mathrm{H}}(\mathrm{t}, \mathrm{s})=\mathrm{E}\left[\beta^{\mathrm{H}}(\mathrm{t}) \beta^{\mathrm{H}}(\mathrm{s})\right]=\frac{1}{2}\left(|\mathrm{t}|^{2 \mathrm{H}}+|\mathrm{s}|^{2 \mathrm{H}}-|\mathrm{t}-\mathrm{s}|^{2 \mathrm{H}}\right), \mathrm{t}, \mathrm{s} \in \mathbb{R}$.

It is known that $\beta^{\mathrm{H}}(\mathrm{t})$ with $\mathrm{H}>\frac{1}{2}$ admits the following Wiener integral representation

$\beta^{\mathrm{H}}(\mathrm{t})=\int_{0}^{\mathrm{t}} \mathrm{K}_{\mathrm{H}}(\mathrm{t}, \mathrm{s}) \mathrm{dW}(\mathrm{s})$

where $\mathrm{W}=\{\mathrm{W}(\mathrm{t}): \mathrm{t} \in[0, \mathrm{~T}]\}$ a Wiener is process and $\mathrm{K}_{\mathrm{H}}(\mathrm{t}, \mathrm{s})$ is the kernel given by

$\mathrm{K}_{\mathrm{H}}(\mathrm{t}, \mathrm{s})=\mathrm{c}_{\mathrm{H}} \int_{\mathrm{s}}^{\mathrm{t}}(\mathrm{u}-\mathrm{s})^{\mathrm{H}-3 / 2}\left(\frac{\mathrm{u}}{\mathrm{s}}\right)^{\mathrm{H}-1 / 2} \mathrm{du}, \mathrm{t}>\mathrm{s}$.

with $\mathrm{c}_{\mathrm{H}}=\sqrt{\frac{\mathrm{H}(2 \mathrm{H}-1)}{\mathrm{B}\left(2-2 \mathrm{H}, \mathrm{H}-\frac{1}{2}\right)}}$ with $\beta(\cdot)$ representing the beta function. Let $\mathrm{K}_{\mathrm{H}}(\mathrm{t}, \mathrm{s})=0$ if $\mathrm{t} \leq \mathrm{s}$.

We will denote by $\mathcal{H}$ the reproducing kernel Hilbert space of the fBm. Infact $\mathcal{H}$ is the closure of set of indicator functions $\left\{1_{[0 ; \mathrm{t}]}, \mathrm{t} \in[0, \mathrm{~T}]\right\}$ with respect to the scalar product

$\left\langle 1_{[0, t]}, 1_{[0, s]}\right\rangle_{\mathcal{H}}=R_{H}(t, s)$.

The mapping $1_{[0, t]} \rightarrow \beta^{\mathrm{H}}(\mathrm{t})$ can be extended to an isometry between $\mathcal{H}$ and the first Wiener chaos and we will denote by $\beta^{\mathrm{H}}(\varphi)$ by the previous isometry. We recall for $\psi, \varphi \in \mathcal{H}$ their scalar product in $\mathcal{H}$ is given by

$$
\langle\psi, \varphi\rangle_{\mathcal{H}}=\mathrm{H}(2 \mathrm{H}-1) \int_{0}^{\mathrm{T}} \int_{0}^{\mathrm{T}} \psi(\mathrm{s}) \varphi(\mathrm{t})|\mathrm{t}-\mathrm{s}|^{2 \mathrm{H}-2} \mathrm{dsdt}
$$

For the deterministic function $\varphi \in \mathrm{L}^{2}([0, \mathrm{~T}])$, the fractional Weiner integral of $\varphi$ with respect to $\beta^{\mathrm{H}}$ is defined by

$\int_{0}^{\mathrm{T}} \varphi(\mathrm{s}) \mathrm{d} \beta^{\mathrm{H}}(\mathrm{t})=\int_{0}^{\mathrm{T}} \mathrm{K}_{\mathrm{H}}^{*} \varphi(\mathrm{s}) \mathrm{d} \beta^{\mathrm{H}}(\mathrm{s})$

where $\mathrm{K}_{\mathrm{H}}^{*} \varphi(\mathrm{s})=\int_{\mathrm{s}}^{\mathrm{T}} \varphi(\mathrm{r}) \frac{\partial \mathrm{k}}{\partial \mathrm{r}}(\mathrm{r}, \mathrm{s}) \mathrm{dr}$ 
Let $\left\{\beta_{\mathrm{n}}^{\mathrm{H}}(\mathrm{t})\right\}_{\mathrm{n} \in \mathbb{N}}$ be a sequence of two-sided one dimensional standard fBm mutually independent on $(\Omega, \mathcal{F}, \mathrm{P})$. When one consider the following series

$$
\sum_{n=1}^{\infty} \beta_{n}^{H}(t) e_{n}, t \geq 0
$$

where $\left\{\mathrm{e}_{\mathrm{n}}\right\}$ is a orthonormal basis in $\mathrm{Y}$, this series does not necessarily converge in the space Y.Thus we consider a $\mathrm{Y}$ - valued stochastic process $\mathrm{B}_{\mathrm{Q}}^{\mathrm{H}}(\mathrm{t})$ given formally by the following series

$$
\mathrm{B}_{\mathrm{Q}}^{\mathrm{H}}(\mathrm{t})=\sum_{\mathrm{n}=1}^{\infty} \beta_{\mathrm{n}}^{\mathrm{H}}(\mathrm{t}) \mathrm{Q}^{\frac{1}{2}} \mathrm{e}_{\mathrm{n},}, \mathrm{t} \geq 0
$$

If $\mathrm{Q}$ is a non-negative self-adjoint trace class operator, then this series converges in the space $\mathrm{Y}$, that is, it holds that $\mathrm{B}_{\mathrm{Q}}^{\mathrm{H}}(\mathrm{t}) \in \mathrm{L}^{2}(\Omega, \mathrm{Y})$ then we say that the above $\mathrm{B}_{\mathrm{Q}}^{\mathrm{H}}(\mathrm{t})$ is a $\mathrm{Y}$ - valued $\mathrm{Q}$ - cylindrical $\mathrm{fBm}$ with covariance operator $\mathrm{Q}$. For example if $\left\{\lambda_{n}\right\}$ is a bounded sequence of non-negative real numbers such that $Q e_{n}=\lambda_{n} e_{n}$ assuming that $Q$ is a nuclear operator in $\mathrm{Y}\left(\mathrm{ie} ., \sum_{\mathrm{n}=1}^{\infty} \lambda_{\mathrm{n}}<\infty\right)$ then the stochastic process

$$
\mathrm{B}_{\mathrm{Q}}^{\mathrm{H}}(\mathrm{t})=\sum_{\mathrm{n}=1}^{\infty} \beta_{\mathrm{n}}^{\mathrm{H}}(\mathrm{t}) \mathrm{Q}^{\frac{1}{2}} \mathrm{e}_{\mathrm{n}}=\sum_{\mathrm{n}=1}^{\infty}{\sqrt{\lambda_{\mathrm{n}}}}^{\mathrm{H}}{ }_{\mathrm{n}}(\mathrm{t}) \mathrm{e}_{\mathrm{n}} \mathrm{t} \geq 0
$$

is well defined as a $\mathrm{Y}$-valued $\mathrm{Q}$ - cylindrical $\mathrm{fBm}$.

where $\beta_{\mathrm{n}}^{\mathrm{H}}(\mathrm{t})$ are real, independent fBm's. This process is a $\mathrm{Y}$-valued Gaussian, it starts from 0 , zero mean and covariance

$E\left\langle\beta^{H}(t), x\right\rangle\left\langle\beta^{H}(s), y\right\rangle=R(s, t)\langle Q(x), y\rangle$ For all $x, y \in Y$ and $t, s \in[0, T]$.

Definition 2.1: Let $\varphi:[0, T] \rightarrow \mathcal{L}_{\mathrm{Q}}^{0}(\mathrm{Y}, \mathrm{X})$ such that $\sum_{\mathrm{n}=1}^{\infty}\left\|\mathrm{K}_{\mathrm{H}}^{*} \varphi \mathrm{Q}^{1 / 2} \mathrm{e}_{\mathrm{n},}\right\|_{\mathrm{L}^{2}([0, \mathrm{~T}]: \mathrm{X})}<\infty$

Then its stochastic integral with respect to the $f B m B_{Q}^{H}$ is defined, for $t \geq 0$, as follows

$$
\int_{0}^{\mathrm{t}} \varphi(s) d B_{\mathrm{Q}}^{\mathrm{H}}(\mathrm{s}):=\sum_{\mathrm{n}=1}^{\infty} \int_{0}^{\mathrm{t}} \varphi(\mathrm{s}) \sqrt{\lambda_{\mathrm{n}}} \mathrm{e}_{\mathrm{n}} \mathrm{d} \beta_{\mathrm{n}}^{\mathrm{H}}(\mathrm{s})=\sum_{\mathrm{n}=1}^{\infty} \int_{0}^{\mathrm{t}} \sqrt{\lambda_{\mathrm{n}}} \mathrm{k}_{\mathrm{H}}^{*}\left(\varphi \mathrm{e}_{\mathrm{n}}\right)(\mathrm{s}) \mathrm{d} \beta_{\mathrm{n}}(\mathrm{s})
$$

Notice that if

$\sum_{\mathrm{n}=1}^{\infty}\left\|\varphi \sqrt{\lambda_{\mathrm{n}}} \mathrm{e}_{\mathrm{n}}\right\|_{\mathrm{L}_{\mathrm{H}}([0, \mathrm{~T}]: \mathrm{H})}<\infty$

Lemma 2.2: For any $\varphi:[0, \mathrm{~T}] \rightarrow \mathcal{L}_{\mathrm{Q}}^{0}(\mathrm{Y}, \mathrm{X})$ satisfies $\int_{0}^{\mathrm{t}}\|\varphi(\mathrm{s})\|_{\mathcal{L}_{\mathrm{Q}}^{0}}^{2} \mathrm{ds}<\infty$ such that $(2.2)$ holds, and for any $\alpha, \beta \in[0, \mathrm{~T}]$ with $\alpha>\beta$,

$$
\mathrm{E}\left\|\int_{\beta}^{\alpha} \varphi(\mathrm{s}) \mathrm{dB}_{\mathrm{Q}}^{\mathrm{H}}(\mathrm{s})\right\|^{2} \leq \mathrm{cH}(2 \mathrm{H}-1)(\alpha-\beta)^{2 \mathrm{H}-1} \sum_{\mathrm{n}=1}^{\infty} \int_{\beta}^{\alpha}\left\|\varphi(\mathrm{s}) \mathrm{Q}^{1 / 2} \mathrm{e}_{\mathrm{n}}\right\|^{2} \mathrm{ds}
$$

where $\mathrm{c}=\mathrm{c}(\mathrm{H})$. If in addition, $\sum_{\mathrm{n}=1}^{\infty}\left\|\varphi(\mathrm{t}) \mathrm{Q}^{1 / 2} \mathrm{e}_{\mathrm{n}}\right\|$ is uniformly convergent for $\mathrm{t} \in[0, \mathrm{~T}]$. Then

$$
\mathrm{E}\left\|\int_{\beta}^{\alpha} \varphi(\mathrm{s}) \mathrm{dB}_{\mathrm{Q}}^{\mathrm{H}}(\mathrm{s})\right\|^{2} \leq \mathrm{cH}(2 \mathrm{H}-1)(\alpha-\beta)^{2 \mathrm{H}-1} \int_{\beta}^{\alpha}\|\varphi(\mathrm{s})\|_{\mathcal{L}_{\mathrm{Q}}^{0}}^{2} \mathrm{ds}
$$

We now suppose that $0 \in \rho(A)$, where $\rho(A)$ is the resolvent set of $A$, and the semigroup $S(t)$ is uniformly bounded that is to say $\|S(t)\| \leq M$ for some constant $M \geq 1$ and every $t \geq 0$. Then, for $0<\alpha \leq 1$, it is possible to define the fractional power operator $(-A)^{\alpha}$ as a closed linear operator on its domain $D(-A)^{\alpha}$. Furthermore the subspace $D(-A)^{\alpha}$ is dense in $\mathrm{X}$ and the expression $\|\mathrm{X}\|_{\alpha}=\left\|(-\mathrm{A})^{\alpha} \mathrm{x}\right\|, \mathrm{x} \in \mathrm{D}(-\mathrm{A})^{\alpha}$ defines a norm on $\mathrm{X}_{\alpha}=\mathrm{D}(-\mathrm{A})^{\alpha}$. The following properties are well known in [18].

Lemma 2.3:[18] Under the above conditions the following properties hold.

i) $\mathrm{X}_{\alpha}$ is a Banach space for $0<\alpha \leq 1$

ii) If the resolvent operator of $\mathrm{A}$ is compact, then embedding $\mathrm{X}_{\beta} \subset \mathrm{X}_{\alpha}$ is continuous and compact for $0<\alpha \leq \beta$.

iii) For every $0<\alpha \leq 1$, there exists $\mathrm{M}_{\alpha}$ such that $\left\|(-\mathrm{A})^{\alpha} \mathrm{S}(\mathrm{t})\right\| \leq \mathrm{M}_{\alpha} \mathrm{t}^{-\alpha} \mathrm{e}^{-\lambda \mathrm{t}}, \lambda>0, t \geq 0$. 
Lemma 2.4:[19] For any $\mathrm{r} \geq 1$ and for arbitrary $\mathrm{L}_{2}^{0}$ valued predictable process $\varphi(\cdot)$,

$$
\sup _{s \in[0, \mathrm{t}]} E\left\|\int_{0}^{s} \varphi(\mathrm{u}) d w(\mathrm{u})\right\|_{\mathrm{X}}^{2 \mathrm{r}} \leq(\mathrm{r}(2 \mathrm{r}-1))^{\mathrm{r}}\left(\int_{0}^{\mathrm{t}}\left(\mathrm{E}\|\varphi(\mathrm{s})\|_{\mathrm{L}_{2}^{0}}^{2 \mathrm{r}}\right)^{\frac{1}{\mathrm{r}}} \mathrm{ds}\right)^{\mathrm{r}}
$$

\section{Existence and uniqueness of a solution}

In this section we study the existence and uniqueness of mild solutions for equation (1.1). For this equation we assume that the following conditions hold.

$\left(\mathrm{H}_{1}\right) \mathrm{A}$ is the infinitesimal generator of an analytic semigroup, $(\mathrm{S}(\mathrm{t}))_{\mathrm{t} \geq 0}$, of bounded linear operators on $X$. Further we suppose that $0 \in \rho(A)$ and that $\|S(t)\| \leq M$ and

$$
\left\|(-A)^{1-\beta} S(t)\right\| \leq \frac{M_{1-\beta}}{t^{1-\beta}} .
$$

For some constants $\mathrm{M}, \mathrm{M}_{1-\beta}$ and every $\mathrm{t} \in[0, \mathrm{~T}]$

$\left(\mathrm{H}_{2}\right)$ There exists a positive constants $\mathrm{C}_{1}, \mathrm{C}_{2}$ and $\mathrm{L}_{1}, \mathrm{~L}_{2}>0$ such that, for all $\mathrm{t} \geq 0, \mathrm{x}, \mathrm{y} \in \mathrm{X}$

i) $\quad\|f(t, x)-f(t, y)\|^{2} \leq L_{1}\|x-y\|^{2}$

ii) $\quad\|\mathrm{f}(\mathrm{t}, \mathrm{x})\|^{2} \leq \mathrm{C}_{1}\left(1+\|\mathrm{x}\|^{2}\right)$

iii) $\quad\|\mathrm{h}(\mathrm{t}, \mathrm{x})-\mathrm{h}(\mathrm{t}, \mathrm{y})\|^{2} \leq \mathrm{L}_{2}\|\mathrm{x}-\mathrm{y}\|^{2}$

iv) $\|\mathrm{h}(\mathrm{t}, \mathrm{x})\|^{2} \leq \mathrm{C}_{2}\left(1+\|\mathrm{x}\|^{2}\right)$

$\left(\mathrm{H}_{3}\right)$ There exists a constant $0<\beta<1, \mathrm{~L}_{3}>0$ such that the function $\mathrm{g}$ is $\mathrm{X}_{\beta}$ - valued and satisfies for all $t \geq 0, x, y \in X$ such that

i) $\quad\left\|(-\mathrm{A})^{\beta} \mathrm{g}(\mathrm{t}, \mathrm{x})-(-\mathrm{A})^{\beta} \mathrm{g}(\mathrm{t}, \mathrm{y})\right\|^{2} \leq \mathrm{L}_{3}\|\mathrm{x}-\mathrm{y}\|^{2}$

ii) $\left\|(-\mathrm{A})^{\beta} \mathrm{g}(\mathrm{t}, \mathrm{x})\right\|^{2} \leq \mathrm{C}_{3}\left(1+\|\mathrm{x}\|^{2}\right)$

iii) The constants $\mathrm{L}_{3}$ and $\beta,\left\|(-\mathrm{A})^{-\beta}\right\| \mathrm{L}_{3}<1$

$\left(\mathrm{H}_{4}\right)$ The function $(-\mathrm{A})^{\beta} \mathrm{g}$ is continuous in the quadratic mean sense square. For all functions $\mathrm{X}$

$$
\lim _{t \rightarrow \infty}\left\|(-A)^{\beta} g(t, x(t))-(-A)^{\beta} g(s, x(s))\right\|^{2}=0
$$

$\left(\mathrm{H}_{5}\right)$ The function $\sigma:[0, \infty) \rightarrow \mathcal{L}_{Q}^{0}(\mathrm{Y}, \mathrm{X})$ satisfies $\int_{0}^{\mathrm{T}}\|\sigma(\mathrm{s})\|_{\mathcal{L}_{\mathrm{Q}}^{0}}^{2} \mathrm{ds}<\infty$,for all $\mathrm{T}>0$.

Moreover we assume that $\phi \in \mathcal{C}\left([-\tau, 0], \mathbb{L}^{2}(\Omega, X)\right)$.

Definition 3.1: A $X$ valued stochastic process $\{x(t), t \in(-\tau, T)\}$ is called a mild solution of Eqn. (1.1) if

i) $\quad \mathrm{x}(\cdot) \in \mathcal{C}\left([-\tau, 0], \mathbb{L}^{2}(\Omega, \mathrm{X})\right)$

ii) $\quad \mathrm{x}(\mathrm{t})=\phi(\mathrm{t})$ for $-\tau \leq \mathrm{t} \leq 0$

iii) For arbitrary $t \in[0, T]$ we have

$$
\begin{aligned}
x(t)= & S(t)(\varphi(0)+g(0, \varphi(-r(0))))-g(t, x(t-r(t))) \\
& -\int_{0}^{t} A S(t-s) g(s, x(s-r(s))) d s+\int_{0}^{t} S(t-s) f(s, x(s-\rho(s))) d s \\
& +\int_{0}^{t} S(t-s) h(s, x(s-\delta(s))) d W(s)+\int_{0}^{t} S(t-s) \sigma(s) d B^{H}(s)
\end{aligned}
$$

Theorem 3.1: Suppose $\left(\mathrm{H}_{1}\right)$ to $\left(\mathrm{H}_{5}\right)$ hold. Then for all $\mathrm{T}>0$, then Eqn. (1.1) has a unique mild solution on $[-\tau, \mathrm{T}]$.

Proof: Fix $\mathrm{T}>0$ and let $\mathcal{B}:=\mathcal{C}[-\tau, \mathrm{T}], \mathbb{L}^{2}(\Omega, \mathrm{X})$, be the Banach space of all continuous functions from $[-\tau, \mathrm{T}]$ into $\mathbb{L}^{2}(\Omega, X)$, equipped with the supremum norm $\|\xi\|_{\mathcal{B}_{\mathrm{T}}}=\sup _{\mathrm{u} \in[-\tau, \mathrm{T}]}\left(\mathrm{E}\|\xi(\mathrm{u})\|^{2}\right)^{1 / 2}$ and let us consider the set $\mathrm{S}_{\mathrm{T}}=$ $\left\{\mathrm{x} \in \mathcal{B}_{\mathrm{T}}: \mathrm{x}(\mathrm{s})=\phi(\mathrm{s})\right.$ for $\left.\mathrm{s} \in[-\tau, 0]\right\}$.

$\mathrm{S}_{\mathrm{T}}$ is a closed subset of $\mathcal{B}_{\mathrm{T}}$ provided with the norm $\|\cdot\|_{\mathcal{B}_{\mathrm{T}}}$. Define the operator $\psi$ on $\mathrm{S}_{\mathrm{T}}$ by $\psi(\mathrm{x})(\mathrm{t})=\phi(\mathrm{t})$ for $\mathrm{t} \in$ $[-\tau, 0]$ and for $\mathrm{t} \in[0, \mathrm{~T}]$ 


$$
\begin{aligned}
\psi(x)(t)= & S(t)(\phi(0)+g(0, \phi(-r(0))))-g(t, x(t-r(t))) \\
& -\int_{0}^{t} A S(t-s) g(s, x(s-r(s))) d s+\int_{0}^{t} S(t-s) f(s, x(s-\rho(s))) d s \\
& +\int_{0}^{t} S(t-s) h(s, x(s-\delta(s))) d W(s)+\int_{0}^{t} S(t-s) \sigma(s) d B^{H}(s)
\end{aligned}
$$

Then it is clear to prove the existence of mild solutions to Eqn. (1.1) is equivalent to find a fixed point for the operator $\psi$. Next we will show by Banach fixed point theorem that $\psi$ has a unique fixed point. We divide the proof into two steps. Step 1 . For arbitrary $\mathrm{x} \in \mathrm{S}_{\mathrm{T}}$, let us prove that $\mathrm{t} \rightarrow \psi(\mathrm{x})(\mathrm{t})$ is continuous on the interval $[0, \mathrm{~T}]$ in the $\mathbb{L}^{2}(\Omega, \mathrm{X})$ sense .Let $0<t<T$ and $|\mathrm{h}|$ be sufficiently small. Then for any $\mathrm{x} \in \mathrm{S}_{\mathrm{T}}$, we have

$$
\begin{aligned}
\|\psi(x)(t+h)-\psi(x)(t)\| & \leq\|(S(t+h)-S(t))(\phi(0)+g(0, \phi(-r(0))))\| \\
& +\|g(t+h, x(t+h-r(t+h)))-g(t, x(t-r(t)))\| \\
& +\left\|\int_{0}^{t+h} A S(t+h-s) g(s, x(s-r(s))) d s-\int_{0}^{t} A S(t-s) g(s, x(s-r(s))) d s\right\| \\
& +\left\|\int_{0}^{t+h} S(t+h-s) f(s, x(s-\rho(s))) d s-\int_{0}^{t} S(t-s) f(s, x(s-\rho(s))) d s\right\| \\
& +\left\|\int_{0}^{t+h} S(t+h-s) h(s, x(s-\delta(s))) d w(s)-\int_{0}^{t} S(t-s) h(s, x(s-\delta(s))) d w(s)\right\| \\
& +\left\|\int_{0}^{t+h} S(t+h-s) \sigma(s) d B^{H}(s)-\int_{0}^{t} S(t-s) \sigma(s) d B^{H}(s)\right\| \\
& =\sum_{1 \leq i \leq 6} I_{i}(h)
\end{aligned}
$$

By the strong continuity of $\mathrm{S}(\mathrm{t})$, we have

$$
\lim _{h \rightarrow 0}\|(S(t+h)-S(t))(\phi(0)+g(0, \phi(-r(0))))\|=0
$$

The condition $\left(\mathrm{H}_{1}\right)$ assure that

$$
\|(\mathrm{S}(\mathrm{t}+\mathrm{h})-\mathrm{S}(\mathrm{t}))(\phi(0)+\mathrm{g}(0, \phi(-\mathrm{r}(0))))\| \leq 2 \mathrm{M}\|\phi(0)+\mathrm{g}(0, \phi(-\mathrm{r}(0)))\| \in \mathbb{L}^{2}(\Omega)
$$

Then we conclude by the Lebesgue dominated theorem that $\lim _{\mathrm{h} \rightarrow 0} \mathbb{E}\left|\mathrm{I}_{1}(\mathrm{~h})\right|=0$

By using the fact that the operator $(-\mathrm{A})^{-\beta}$ is bounded, we obtained that

$$
\mathbb{E}\left|I_{2}(h)\right|^{2} \leq\left\|(-A)^{-\beta}\right\|^{2} \mathbb{E}\left\|(-A)^{\beta} g(t+h, x(t+h-r(t+h)))-(-A)^{\beta} g(t, x(t-r(t)))\right\|^{2}
$$

Then we conclude by condition $\left(\mathrm{H}_{4}\right)$ that $\lim _{\mathrm{h} \rightarrow 0} \mathbb{E}\left|\mathrm{I}_{2}(\mathrm{~h})\right|=0$

For $I_{3}(h)$, we suppose that $h>0$, then we have

$$
\begin{aligned}
I_{3}(h) \leq & \left\|\int_{0}^{t} S((h)-I)(-A)^{1-\beta} S(t-s)(-A)^{\beta} g(s, x(s-r(s))) d s\right\| \\
& +\left\|\int_{t}^{t+h}(-A)^{1-\beta} S(t+h-s)(-A)^{\beta} g(s, x(s-r(s))) d s\right\|
\end{aligned}
$$

By Holder's inequality

$$
\begin{aligned}
\mathbb{E}\left\|\int_{0}^{t} S((h)-I)(-A)^{1-\beta} S(t-s)(-A)^{\beta} g(s, x(s-r(s))) d s\right\|^{2} & \\
& \leq t \mathbb{E} \int_{0}^{t}\left\|S((h)-I)(-A)^{1-\beta} S(t-s)(-A)^{\beta} g(s, x(s-r(s)))\right\|^{2} d s
\end{aligned}
$$


By using the strong continuity of $S(t)$, we have for each $s \in[0, t]$,

$$
\lim _{h \rightarrow 0} S((h)-I)(-A)^{1-\beta} S(t-s)(-A)^{\beta} g(s, x(s-r(s)))=0
$$

By using condition $\left(\mathrm{H}_{1}\right)$, condition (ii) in $\left(\mathrm{H}_{3}\right)$ and the fact that $0<\beta<1$, we obtain

$$
\begin{aligned}
& \left\|S((h)-I)(-A)^{1-\beta} S(t-s)(-A)^{\beta} g(s, x(s-r(s)))\right\| \\
& \quad \leq \frac{(M+1) M_{1-\beta}}{(t-s)^{1-\beta}}\left\|(-A)^{\beta} g(s, x(s-r(s)))\right\| \in \mathbb{L}^{2}([0, t] \times \Omega)
\end{aligned}
$$

Then we conclude by the Lebesgue dominated theorem that

$$
\lim _{h \rightarrow 0} \mathbb{E}\left\|\int_{0}^{t} S((h)-I)(-A)^{1-\beta} S(t-s)(-A)^{\beta} g(s, x(s-r(s))) d s\right\|^{2}=0
$$

By conditions $\left(\mathrm{H}_{1}\right)$, condition (ii)in $\left(\mathrm{H}_{3}\right)$ and Holder's inequality we get

$$
\begin{array}{r}
\mathbb{E}\left\|\int_{t}^{\mathrm{t}+\mathrm{h}}(-\mathrm{A})^{1-\beta} \mathrm{S}(\mathrm{t}+\mathrm{h}-\mathrm{s})(-\mathrm{A})^{\beta} \mathrm{g}(\mathrm{s}, \mathrm{x}(\mathrm{s}-\mathrm{r}(\mathrm{s}))) \mathrm{ds}\right\|^{2} \\
\quad \leq \frac{\mathrm{M}^{2}{ }_{1-\beta}}{2 \beta-1} \mathrm{~h}^{2 \beta-1} \int_{0}^{\mathrm{T}} \mathrm{C}_{3}^{2}\left(\mathbb{E}\|\mathrm{x}(\mathrm{s})-\mathrm{r}(\mathrm{s})\|^{2}+1\right) \mathrm{ds}
\end{array}
$$

Then $\lim _{\mathrm{h} \rightarrow 0}\left\|\int_{\mathrm{t}}^{\mathrm{t}+\mathrm{h}}(-\mathrm{A})^{1-\beta} \mathrm{S}(\mathrm{t}+\mathrm{h}-\mathrm{s})(-\mathrm{A})^{\beta} \mathrm{g}(\mathrm{s}, \mathrm{x}(\mathrm{s}-\mathrm{r}(\mathrm{s}))) \mathrm{ds}\right\|^{2}=0$

Hence that $\lim _{\mathrm{h} \rightarrow 0} \mathbb{E}\left|\mathrm{I}_{3}(\mathrm{~h})\right|=0$

$$
\begin{array}{r}
I_{4}(h) \leq\left\|\int_{0}^{t} S((h)-I) S(t-s) f(s, x(s-\rho(s))) d s\right\| \\
+\left\|\int_{t}^{t+h} S(t+h-s) f(s, x(s-\rho(s))) d s\right\|
\end{array}
$$

Now $\mathbb{E}\left\|\int_{0}^{t} S((h)-I) S(t-s) f(s, x(s-\rho(s))) d s\right\|^{2}$

$$
\begin{aligned}
& \leq \int_{0}^{\mathrm{t}} \mathrm{S}((\mathrm{h})-\mathrm{I}) \mathrm{S}(\mathrm{t}-\mathrm{s}) \mathrm{C}_{1}^{2}\left(\mathbb{E}\|\mathrm{x}(\mathrm{s}-\rho(\mathrm{s}))\|^{2}+1\right) \mathrm{ds} \\
& \leq \mathrm{M} \int_{0}^{\mathrm{T}} \mathrm{S}((\mathrm{h})-\mathrm{I}) \mathrm{C}_{1}^{2} \mathbb{E}\left(\|\mathrm{x}(\mathrm{s}-\rho(\mathrm{s}))\|^{2}+1\right) \mathrm{ds}
\end{aligned}
$$

Since $\lim _{h \rightarrow 0} \mathbb{E}\left\|\int_{0}^{t} S((h)-I) S(t-s) f(s, x(s-\rho(s))) d s\right\|^{2}=0$

and $\mathbb{E}\left\|\int_{t}^{t+h} S(t+h-s) f(s, x(s-\rho(s))) d s\right\|^{2}$

$$
\begin{aligned}
& \leq M^{2} \mathbb{E}\left\|\int_{t}^{t+h} f(s, x(s-\rho(s))) d s\right\|^{2} \\
& \leq M^{2} \int_{t}^{t+h} C_{1}^{2} \mathbb{E}\left(\|x(s-\rho(s))\|^{2}+1\right) d s
\end{aligned}
$$

Then $\lim _{h \rightarrow 0} \mathbb{E}\left\|\int_{t}^{t+h} S(t+h-s) f(s, x(s-\rho(s))) d s\right\|^{2}=0$

Hence $\lim _{\mathrm{h} \rightarrow 0} \mathbb{E}\left|\mathrm{I}_{4}(\mathrm{~h})\right|=0$

Now $I_{5}(h) \leq\left\|\int_{0}^{t} S((h)-I) S(t-s) h(s, x(s-\delta(s))) d W(s)\right\|$ 


$$
+\left\|\int_{t}^{t+h} S(t+h-s) h(s, x(s-\delta(s))) d W(s)\right\|
$$

Now $\mathbb{E}\left\|\int_{0}^{t} S((h)-I) S(t-s) h(s, x(s-\delta(s))) d W(s)\right\|^{2}$

$$
\begin{aligned}
& \leq \int_{0}^{\mathrm{t}} \mathrm{S}((\mathrm{h})-\mathrm{I}) \mathrm{S}(\mathrm{t}-\mathrm{s}) \mathrm{C}_{2}^{2}\left(\mathbb{E}\|\mathrm{x}(\mathrm{s}-\delta(\mathrm{s}))\|^{2}+1\right) \mathrm{ds} \\
& \leq \mathrm{M}^{2} \int_{0}^{\mathrm{t}} \mathrm{S}((\mathrm{h})-\mathrm{I}) \mathrm{C}_{2}^{2}\left(\mathbb{E}\|\mathrm{x}(\mathrm{s}-\delta(\mathrm{s}))\|^{2}+1\right) \mathrm{ds}
\end{aligned}
$$

Therefore $\lim _{h \rightarrow 0} \mathbb{E}\left\|\int_{0}^{t} S((h)-I) S(t-s) h(s, x(s-\delta(s))) d W(s)\right\|^{2}=0$

and $\mathbb{E}\left\|\int_{\mathrm{t}}^{\mathrm{t}+\mathrm{h}} \mathrm{S}(\mathrm{t}+\mathrm{h}-\mathrm{s}) \mathrm{h}(\mathrm{s}, \mathrm{x}(\mathrm{s}-\delta(\mathrm{s}))) \mathrm{dW}(\mathrm{s})\right\|^{2}$

$$
\leq \mathrm{M}^{2} \int_{\mathrm{t}}^{\mathrm{t}+\mathrm{h}} \mathrm{C}_{2}^{2} \mathbb{E}\left(\|\mathrm{x}(\mathrm{s}-\delta(\mathrm{s}))\|^{2}+1\right) \mathrm{ds}
$$

Then $\lim _{h \rightarrow 0} \mathbb{E}\left\|\int_{t}^{t+h} S(t+h-s) h(s, x(s-\delta(s))) d W(s)\right\|^{2}=0$

Hence $\lim _{\mathrm{h} \rightarrow 0} \mathbb{E}\left|I_{5}(\mathrm{~h})\right|=0$

For the term $\mathrm{I}_{6}(\mathrm{~h})$, we have

$$
\mathrm{I}_{6}(\mathrm{~h}) \leq\left\|\int_{0}^{\mathrm{t}} \mathrm{S}((\mathrm{h})-\mathrm{I}) \mathrm{S}(\mathrm{t}-\mathrm{s}) \sigma(\mathrm{s}) \mathrm{dB}^{\mathrm{H}}(\mathrm{s})\right\|+\left\|\int_{\mathrm{t}}^{\mathrm{t}+\mathrm{h}} \mathrm{S}(\mathrm{t}+\mathrm{h}-\mathrm{s}) \sigma(\mathrm{s}) \mathrm{dB}^{\mathrm{H}}(\mathrm{s})\right\|
$$

By condition $\left(\mathrm{H}_{1}\right)$ and lemma 2.2 we get

$$
\begin{aligned}
& \mathbb{E}\left\|\int_{0}^{\mathrm{t}} \mathrm{S}((\mathrm{h})-\mathrm{I}) \mathrm{S}(\mathrm{t}-\mathrm{s}) \sigma(\mathrm{s}) \mathrm{dB}^{\mathrm{H}}(\mathrm{s})\right\|^{2} \\
& \quad \leq \mathrm{cH}(2 \mathrm{H}-1)(\mathrm{t})^{2 \mathrm{H}-1} \int_{0}^{\mathrm{t}}\|\mathrm{S}((\mathrm{h})-\mathrm{I}) \mathrm{S}(\mathrm{t}-\mathrm{s}) \sigma(\mathrm{s})\|_{\mathcal{L}_{\mathrm{Q}}^{0}}^{2} \mathrm{ds} \\
& \quad \leq \mathrm{cH}(2 \mathrm{H}-1)(\mathrm{t})^{2 \mathrm{H}-1} \mathrm{M} \int_{0}^{\mathrm{T}}\|\mathrm{S}((\mathrm{h})-\mathrm{I}) \sigma(\mathrm{s})\|_{\mathcal{L}_{\mathrm{Q}}^{0}}^{2} \mathrm{ds}
\end{aligned}
$$

Since $\lim _{\mathrm{h} \rightarrow 0}\|S((\mathrm{~h})-\mathrm{I}) \sigma(\mathrm{s})\|_{\mathcal{L}_{\mathrm{Q}}^{0}}^{2}=0$ and

$$
\|\mathrm{S}((\mathrm{h})-\mathrm{I}) \sigma(\mathrm{s})\|_{\mathcal{L}_{\mathrm{Q}}^{0}}^{2} \leq 4 \mathrm{M}^{2}\|\sigma(\mathrm{s})\|_{\mathcal{L}_{\mathrm{Q}}^{0}}^{2} \in \mathcal{L}^{1}([0, \mathrm{~T}], \mathrm{ds})
$$

we conclude by the dominated convergence theorem that

$\lim _{h \rightarrow 0} \mathbb{E}\left\|\int_{0}^{t} S((h)-I) S(t-s) \sigma(s) d B^{H}(s)\right\|^{2}=0$

Applying lemma 2.2, we get

$$
\mathbb{E}\left\|\int_{\mathrm{t}}^{\mathrm{t}+\mathrm{h}} \mathrm{S}(\mathrm{t}+\mathrm{h}-\mathrm{s}) \sigma(\mathrm{s}) \mathrm{dB}^{\mathrm{H}}(\mathrm{s})\right\| \leq \mathrm{cH}(\mathrm{h})^{2 \mathrm{H}-1} \mathrm{M}^{2} \int_{\mathrm{t}}^{\mathrm{t}+\mathrm{h}}\|\sigma(\mathrm{s})\|_{\mathcal{L}_{\mathrm{Q}}^{0}}^{2} \mathrm{ds} \rightarrow 0
$$

Hence that $\lim _{\mathrm{h} \rightarrow 0} \mathbb{E}\left|\mathrm{I}_{6}(\mathrm{~h})\right|=0$

The above arguments show that $\lim _{\mathrm{h} \rightarrow 0} \mathbb{E}\|\psi(\mathrm{x})(\mathrm{t}+\mathrm{h})-\psi(\mathrm{x})(\mathrm{t})\|^{2}=0$. Hence we conclude that the function $\mathrm{t} \rightarrow \psi(\mathrm{x})(\mathrm{t})$ is continuous on $[0, T]$ in the $\mathbb{L}^{2}$ - sense.

Step 2. Now we are going to show that $\psi$ is a contraction mapping in $S_{T_{1}}$ with some $T_{1} \leq T$.

Let $x, y \in S_{T}$ by using the inequality $(a+b+c+d)^{2} \leq \frac{1}{k} a^{2}+\frac{3}{1-k} b^{2}+\frac{3}{1-k} c^{2}+\frac{3}{1-k} d^{2}$

where $\mathrm{k}=\mathrm{L}_{3}\left\|(-\mathrm{A})^{-\beta}\right\|<1$, we obtain any fixed $\mathrm{t} \in[0, \mathrm{~T}]$

$\mathbb{E}\|\psi(\mathrm{x})(\mathrm{t})-\psi(\mathrm{y})(\mathrm{t})\|^{2} \leq \frac{1}{\mathrm{k}}\|\mathrm{g}(\mathrm{t}, \mathrm{x}(\mathrm{t}-\mathrm{r}(\mathrm{t})))-\mathrm{g}(\mathrm{t}, \mathrm{y}(\mathrm{t}-\mathrm{r}(\mathrm{t})))\|^{2}$ 


$$
\begin{aligned}
& +\frac{3}{1-\mathrm{k}}\left\|\int_{0}^{\mathrm{t}} \mathrm{AS}(\mathrm{t}-\mathrm{s})(\mathrm{g}(\mathrm{s}, \mathrm{x}(\mathrm{s}-\mathrm{r}(\mathrm{s})))-\mathrm{g}(\mathrm{s}, \mathrm{y}(\mathrm{s}-\mathrm{r}(\mathrm{s})))) \mathrm{ds}\right\|^{2} \\
& +\frac{3}{1-\mathrm{k}}\left\|\int_{0}^{\mathrm{t}} \mathrm{S}(\mathrm{t}-\mathrm{s})(\mathrm{f}(\mathrm{s}, \mathrm{x}(\mathrm{s}-\rho(\mathrm{s})))-\mathrm{f}(\mathrm{s}, \mathrm{y}(\mathrm{s}-\rho(\mathrm{s})))) \mathrm{ds}\right\|^{2} \\
& +\frac{3}{1-\mathrm{k}}\left\|\int_{0}^{\mathrm{t}} \mathrm{S}(\mathrm{t}-\mathrm{s})(\mathrm{h}(\mathrm{s}, \mathrm{x}(\mathrm{s}-\delta(\mathrm{s})))-\mathrm{h}(\mathrm{s}, \mathrm{y}(\mathrm{s}-\delta(\mathrm{s})))) \mathrm{dW}(\mathrm{s})\right\|^{2} \\
& \leq \frac{1}{\mathrm{k}}\left\|(-\mathrm{A})^{-\beta}\right\|^{2}\left\|(-\mathrm{A})^{\beta} \mathrm{g}(\mathrm{t}, \mathrm{x}(\mathrm{t}-\mathrm{r}(\mathrm{t})))-(-\mathrm{A})^{\beta} \mathrm{g}(\mathrm{t}, \mathrm{y}(\mathrm{t}-\mathrm{r}(\mathrm{t})))\right\|^{2} \\
& +\frac{3}{1-\mathrm{k}}\left\|\int_{0}^{\mathrm{t}}(-\mathrm{A})^{1-\beta} \mathrm{S}(\mathrm{t}-\mathrm{s})(-\mathrm{A})^{\beta}(\mathrm{g}(\mathrm{s}, \mathrm{x}(\mathrm{s}-\mathrm{r}(\mathrm{s})))-\mathrm{g}(\mathrm{s}, \mathrm{y}(\mathrm{s}-\mathrm{r}(\mathrm{s}))))\right\|^{2} \\
& +\frac{3}{1-\mathrm{k}}\left\|\int_{0}^{\mathrm{t}} \mathrm{S}(\mathrm{t}-\mathrm{s})(-\mathrm{A})^{\beta}(\mathrm{f}(\mathrm{s}, \mathrm{x}(\mathrm{s}-\rho(\mathrm{s})))-\mathrm{f}(\mathrm{s}, \mathrm{y}(\mathrm{s}-\rho(\mathrm{s})))) \mathrm{ds}\right\|^{2} \\
& +\frac{3}{1-\mathrm{k}}\left\|\int_{0}^{\mathrm{t}} \mathrm{S}(\mathrm{t}-\mathrm{s})(-\mathrm{A})^{\beta}(\mathrm{h}(\mathrm{s}, \mathrm{x}(\mathrm{s}-\delta(\mathrm{s})))-\mathrm{h}(\mathrm{s}, \mathrm{y}(\mathrm{s}-\delta(\mathrm{s})))) \mathrm{dW}(\mathrm{s})\right\|^{2}
\end{aligned}
$$

By Lipchitz property of $(-\mathrm{A})^{\beta} \mathrm{g}$ and $\mathrm{f}, \mathrm{h}$ combined with Holder's inequality, we obtain

$\mathbb{E}\|\psi(\mathrm{x})(\mathrm{t})-\psi(\mathrm{y})(\mathrm{t})\|^{2} \leq \mathrm{k} \mathbb{E}\|\mathrm{x}(\mathrm{t}-\mathrm{r})-\mathrm{y}(\mathrm{t}-\mathrm{r})\|^{2}$

$$
\begin{aligned}
& +\frac{3}{1-\mathrm{k}} \mathrm{C}_{3}^{2} \mathrm{M}_{1-\beta}^{2}\left(\frac{\mathrm{t}^{2 \beta-1}}{2 \beta-1}\right) \int_{0}^{\mathrm{t}} \mathbb{E}\|\mathrm{x}(\mathrm{s}-\mathrm{r})-\mathrm{y}(\mathrm{s}-\mathrm{r})\|^{2} \mathrm{ds} \\
& +\frac{3}{1-\mathrm{k}} \mathrm{tM}^{2} \mathrm{C}_{1}^{2} \int_{0}^{\mathrm{t}} \mathbb{E}\|\mathrm{x}(\mathrm{s}-\rho(\mathrm{s}))-\mathrm{y}(\mathrm{s}-\rho(\mathrm{s}))\|^{2} \mathrm{ds} \\
& +\frac{3}{1-\mathrm{k}} \mathrm{tM}^{2} \mathrm{C}_{2}^{2} \int_{0}^{\mathrm{t}} \mathbb{E}\|\mathrm{x}(\mathrm{s}-\delta(\mathrm{s}))-\mathrm{y}(\mathrm{s}-\delta(\mathrm{s}))\|^{2} \mathrm{ds}
\end{aligned}
$$

Hencesup $_{\mathrm{s} \in[-\tau, \mathrm{t}]} \mathbb{E}\|\psi(\mathrm{x})(\mathrm{s})-\psi(\mathrm{y})(\mathrm{s})\|^{2} \leq \gamma(\mathrm{t}) \sup _{\mathrm{s} \in[-\tau, \mathrm{t}]} \mathbb{E}\|\mathrm{x}(\mathrm{s})-\mathrm{y}(\mathrm{s})\|^{2}$

where $\gamma(\mathrm{t})=\mathrm{k}+\frac{3 \mathrm{C}_{3}^{2} \mathrm{M}_{1-\beta}^{2}}{(1-\mathrm{k})(2 \beta-1)} \mathrm{t}^{2 \beta}+\frac{3 \mathrm{M}^{2} \mathrm{C}_{1}^{2} \mathrm{t}^{2}}{1-\mathrm{k}}+\frac{3 \mathrm{M}^{2} \mathrm{C}^{2} \mathrm{t}^{2}}{1-\mathrm{k}}$

By condition (iii)in $\left(\mathrm{H}_{3}\right)$ we have, $\gamma(0)=\mathrm{k}=\left\|(-\mathrm{A})^{-\beta}\right\| \mathrm{L}_{3}<1$.Then there exists $0<T_{1} \leq \mathrm{T}$ such that $0<\gamma\left(\mathrm{T}_{1}\right)<1$ and $\psi$ is a contraction mapping on $\mathrm{S}_{\mathrm{T}_{1}}$ and therefore has a unique fixed point, which is a mild solution of Eqn.(1.1) on $\left[-\tau, \mathrm{T}_{1}\right]$.This procedure can be repeated in order to extend the solution to the entire interval $[-\tau, \mathrm{T}]$ in finitely many steps. This completes the proof.

\section{Stability analysis}

In this section we establish the results for the case of finite delays, then the case of infinite delays can be proved. Our method is based on the contraction mapping principle.

In order to prove the required results, we assume the following additional conditions.

$\left(\mathrm{H}_{6}\right)$ The semigroup $\mathrm{S}(\mathrm{t})$ satisfies $\exists \lambda>0, \exists \mathrm{M}>0$ such that $\|\mathrm{S}(\mathrm{t})\| \leq \mathrm{Me}^{-\lambda \mathrm{t}}, \mathrm{t} \geq 0$

$\left(\mathrm{H}_{7}\right)$ The function $\sigma:[0, \infty) \rightarrow \mathcal{L}_{Q}^{0}$ satisfies $\int_{0}^{\infty} \mathrm{e}^{\gamma s}\|\sigma(\mathrm{s})\|_{\mathcal{L}_{Q}^{0}}^{2} \mathrm{ds}<\infty$.

We first consider the case of finite delays, $\tau<\infty$.

Theorem 4.1: (Finite delays) Assume that $\mathrm{f}(\mathrm{t}, 0)=\mathrm{g}(\mathrm{t}, 0)=\mathrm{h}(\mathrm{t}, 0)=0, \mathrm{t} \geq 0$, the assumptions $\left(\mathrm{H}_{1}\right)-\left(\mathrm{H}_{7}\right)$ holds and that

$$
4\left(\mathrm{~L}_{3}\left\|(-\mathrm{A})^{-\beta}\right\|^{2}+\mathrm{M}_{1-\beta}^{2} \mathrm{~L}_{3} \frac{\Gamma^{2}(\beta)}{\lambda^{2 \beta}}+\mathrm{M}^{2} \mathrm{~L}_{1} \lambda^{-2}+\mathrm{M}^{2} \mathrm{~L}_{2}(2 \lambda)^{-2}\right)<1
$$


where $\Gamma(\cdot)$ is the gamma function, $\mathrm{M}_{1-\beta}$ is the corresponding constant in lemma (2.3). Then the mild solution to (1.1) exists uniquely and is exponential decay to zero in mean square. i.e., there exists a pair of positive constants a $>0$ and $M^{*}=M^{*}(\varphi, a)$ such that

$$
\mathbb{E}\|\mathrm{x}(\mathrm{t})\|^{2} \leq \mathrm{M}^{*} \mathrm{e}^{-\mathrm{at}}, \forall \mathrm{t} \geq 0
$$

Proof: Denote by $s$ the space of all stochastic process $x(t, \omega):(-\tau, \infty) \times \Omega \rightarrow X$ satisfying $x(t)=\phi(t), t \in(-\tau, 0]$ and there exists some constants $\mathrm{a}>0$ and $\mathrm{M}^{*}=\mathrm{M}^{*}(\varphi, \mathrm{a})>0$ such that

$$
\mathbb{E}\|\mathrm{x}(\mathrm{t})\|^{2} \leq \mathrm{M}^{*} \mathrm{e}^{-\mathrm{at}}, \forall \mathrm{t} \geq 0
$$

It is to check that $s$ is a Banach space endowed with a norm $|\mathrm{x}|_{s}^{2}=\sup _{\mathrm{t} \geq 0} \mathbb{E}|\mathrm{x}(\mathrm{t})|^{2}$. Without loss of generality, we assume that $\mathrm{a}<\lambda$. We denote the operator $\Phi$ on $s$ by

$$
\begin{aligned}
& (\Phi \mathrm{x})(\mathrm{t})=\phi(\mathrm{t}), \mathrm{t} \in(-\tau, 0] \text { and } \\
& (\Phi \mathrm{x})(\mathrm{t})=\mathrm{S}(\mathrm{t})(\phi(0)+\mathrm{g}(0, \phi(-\mathrm{r}(0))))-\mathrm{g}(\mathrm{t}, \mathrm{x}(\mathrm{t}-\mathrm{r}(\mathrm{t}))) \\
& -\int_{0}^{t} \operatorname{AS}(t-s) g(s, x(s-r(s))) d s+\int_{0}^{t} S(t-s) f(s, x(s-\rho(s))) d s \\
& +\int_{0}^{t} \mathrm{~S}(\mathrm{t}-\mathrm{s}) \mathrm{h}(\mathrm{s}, \mathrm{x}(\mathrm{s}-\delta(\mathrm{s}))) d W(\mathrm{~s})+\int_{0}^{\mathrm{t}} \mathrm{S}(\mathrm{t}-\mathrm{s}) \sigma(\mathrm{s}) \mathrm{dB}^{\mathrm{H}}(\mathrm{s})
\end{aligned}
$$

To get desired results, it is enough to show that operator $\Phi$ has a unique fixed point in 8 . For this purpose, we use the contraction mapping principle.

Step 1 . We first verify that $\Phi(s) \subset s$. For convenience of notation, we denote by $M_{i}^{*}, i=1,2, \ldots$ the finite positive constants depending on $\varphi$, a. By assumption $\left(\mathrm{H}_{6}\right)$ we have

$$
\mathbb{E}\left\|p_{1}(t)\right\|^{2} \leq M^{2} \mathbb{E}\|\phi(0)+g(0, \phi(-r(0)))\|^{2} e^{-\lambda t} \leq M_{1}^{*} e^{-\lambda t}
$$

To estimate $\mathrm{p}_{\mathrm{i}}(\mathrm{t}), \mathrm{i}=2, \ldots, 6$. we observe that for $\mathrm{x} \in \mathrm{s}$ and $\mathrm{u}(\mathrm{t})=\mathrm{r}(\mathrm{t})$ or $\rho(\mathrm{t})$ or $\delta(\mathrm{t})$ the following useful estimate holds

$$
\begin{aligned}
\mathbb{E}\|\mathrm{x}(\mathrm{t}-\mathrm{u}(\mathrm{t}))\|^{2} & \leq\left(\mathrm{M}^{*} \mathrm{e}^{-\mathrm{a}(\mathrm{t}-\mathrm{u}(\mathrm{t}))}+\mathbb{E}\|\varphi(\mathrm{t}-\mathrm{u}(\mathrm{t}))\|^{2}\right) \\
& \leq\left(\mathrm{M}^{*} \mathrm{e}^{-\mathrm{a}(\mathrm{t}-\mathrm{u}(\mathrm{t}))}+\mathbb{E} \mathrm{E}\|\varphi\|_{\mathrm{c}}^{2} \mathrm{e}^{-\mathrm{a}(\mathrm{t}-\mathrm{u}(\mathrm{t}))}\right) \\
& \leq\left(\mathrm{M}^{*}+\mathbb{E}\|\phi\|_{\mathrm{c}}^{2}\right) \mathrm{e}^{\mathrm{a} \tau} \mathrm{e}^{-\mathrm{at}}
\end{aligned}
$$

where $\|\phi\|_{c}=\sup _{-\tau<s \leq 0}\|\varphi(s)\|<\infty$.

Then by assumption (i) in $\left(\mathrm{H}_{3}\right)$ we have

$$
\begin{aligned}
\mathbb{E}\left\|p_{2}(\mathrm{t})\right\|^{2} & \leq\left\|(-\mathrm{A})^{-\beta}\right\|^{2} \mathbb{E}\left\|(-\mathrm{A})^{\beta} \mathrm{g}(\mathrm{t}, \mathrm{x}(\mathrm{t}-\mathrm{r}(\mathrm{t})))-(-\mathrm{A})^{\beta} \mathrm{g}(\mathrm{t}, 0)\right\|^{2} \\
& \leq \mathrm{L}_{3}\left\|(-\mathrm{A})^{-\beta}\right\|^{2} \mathbb{E}\|\mathrm{x}(\mathrm{t}-\mathrm{r}(\mathrm{t}))\|^{2} \\
& \leq \mathrm{L}_{3}\left\|(-\mathrm{A})^{-\beta}\right\|^{2}\left(\mathrm{M}^{*}+\mathbb{E}\|\phi\|_{\mathrm{c}}^{2}\right) \mathrm{e}^{\mathrm{a} \tau} \mathrm{e}^{-\mathrm{at}} \\
& \leq \mathrm{M}_{2}^{*} \mathrm{e}^{-\mathrm{at}}
\end{aligned}
$$

Using lemma 2.3, Holder's inequality and assumption (i) in $\left(\mathrm{H}_{3}\right)$ obtain that

$$
\begin{aligned}
\mathbb{E}\left\|p_{3}(t)\right\|^{2} & \leq \mathbb{E}\left\|\int_{0}^{t} A S(t-s) g(s, x(s-r(s))) d s\right\|^{2} \\
& \leq\left\|(-A)^{1-\beta} S(t-s)\right\| d s \int_{0}^{t}\left\|(-A)^{1-\beta} S(t-s)\right\| \mathbb{E}\left\|(-A)^{\beta} g(s, x(s-r(s)))\right\|^{2} d s
\end{aligned}
$$




$$
\begin{aligned}
& \leq \mathrm{M}_{1-\beta}^{2} \mathrm{~L}_{3} \int_{0}^{\mathrm{t}}(\mathrm{t}-\mathrm{s})^{\beta-1} \mathrm{e}^{-\lambda(\mathrm{t}-\mathrm{s})} \mathrm{ds} \int_{0}^{\mathrm{t}}(\mathrm{t}-\mathrm{s})^{\beta-1} \mathrm{e}^{-\lambda(\mathrm{t}-\mathrm{s})} \mathbb{E}\|\mathrm{x}(\mathrm{s}-\mathrm{r}(\mathrm{s}))\|^{2} \mathrm{ds} \\
& \leq \mathrm{M}_{1-\beta}^{2} \mathrm{~L}_{3} \frac{\Gamma(\beta)}{\lambda^{\beta}} \int_{0}^{\mathrm{t}}(\mathrm{t}-\mathrm{s})^{\beta-1} \mathrm{e}^{-\lambda(\mathrm{t}-\mathrm{s})}\left(\mathrm{M}^{*}+\mathbb{E}\|\varphi\|_{\mathrm{c}}^{2}\right) \mathrm{e}^{\mathrm{a} \tau} \mathrm{e}^{-\mathrm{as}} \mathrm{ds} \\
& \leq \mathrm{M}_{1-\beta}^{2} \mathrm{~L}_{3} \frac{\Gamma(\beta)}{\lambda^{\beta}}\left(\mathrm{M}^{*}+\mathbb{E}\|\varphi\|_{\mathrm{c}}^{2}\right) \mathrm{e}^{\mathrm{a} \tau} \mathrm{e}^{-\mathrm{at}} \int_{0}^{\mathrm{t}}(\mathrm{t}-\mathrm{s})^{\beta-1} \mathrm{e}^{(\mathrm{a}-\lambda)(\mathrm{t}-\mathrm{s})} \mathrm{ds} \\
& \leq \mathrm{M}_{1-\beta}^{2} \mathrm{~L}_{3} \frac{\Gamma^{2}(\beta)}{\lambda^{\beta}(\lambda-\mathrm{a})^{\beta}}\left(\mathrm{M}^{*}+\mathbb{E}\|\phi\|_{\mathrm{c}}^{2}\right) \mathrm{e}^{\mathrm{a} \tau} \mathrm{e}^{-\mathrm{at}}
\end{aligned}
$$

We therefore have $\mathbb{E}\left\|p_{3}(t)\right\|^{2} \leq M_{3}^{*} e^{-a t}$

Similarly, we obtain by assumption(i) in $\left(\mathrm{H}_{2}\right)$ that

$$
\begin{gathered}
\mathbb{E}\left\|p_{4}(\mathrm{t})\right\|^{2}=\mathbb{E}\left\|\int_{0}^{\mathrm{t}} \mathrm{S}(\mathrm{t}-\mathrm{s}) \mathrm{f}(\mathrm{s}, \mathrm{x}(\mathrm{s}-\rho(\mathrm{s}))) \mathrm{ds}\right\|^{2} \\
\leq \mathrm{M}^{2} \mathrm{~L}_{1} \int_{0}^{\mathrm{t}} \mathrm{e}^{-\lambda(\mathrm{t}-\mathrm{s})} \mathrm{ds} \int_{0}^{\mathrm{t}} \mathrm{e}^{-\lambda(\mathrm{t}-\mathrm{s})} \mathbb{E}\|\mathrm{x}(\mathrm{s}-\rho(\mathrm{s}))\|^{2} \mathrm{ds} \\
\leq \mathrm{M}^{2} \mathrm{~L}_{1} \lambda^{-1} \int_{0}^{\mathrm{t}} \mathrm{e}^{-\lambda(\mathrm{t}-\mathrm{s})}\left(\mathrm{M}^{*}+\mathbb{E}\|\phi\|_{\mathrm{c}}^{2}\right) \mathrm{e}^{\mathrm{a} \tau} \mathrm{e}^{-\mathrm{as}} \mathrm{ds} \\
\leq \mathrm{M}^{2} \mathrm{~L}_{1} \lambda^{-1}(\lambda-\mathrm{a})^{-1}\left(\mathrm{M}^{*}+\mathbb{E}\|\phi\|_{\mathrm{c}}^{2}\right) \mathrm{e}^{\mathrm{a} \tau} \mathrm{e}^{-\mathrm{at}} \\
\leq \mathrm{M}_{4}^{*} \mathrm{e}^{-\mathrm{at}} \\
\mathbb{E}\left\|\mathrm{p}_{5}(\mathrm{t})\right\|^{2}=\mathbb{E}\left\|\int_{0}^{\mathrm{t}} \mathrm{S}(\mathrm{t}-\mathrm{s}) \mathrm{h}(\mathrm{s}, \mathrm{x}(\mathrm{s}-\delta(\mathrm{s}))) \mathrm{dw}(\mathrm{s})\right\|^{2} \\
\leq \mathrm{M}^{2} \mathrm{~L}_{2} \int_{0}^{\mathrm{t}} \mathrm{e}^{-2 \lambda(\mathrm{t}-\mathrm{s})} \mathbb{E}\|\mathrm{x}(\mathrm{s}-\delta(\mathrm{s}))\|^{2} \mathrm{ds} \\
\leq \mathrm{M}^{2} \mathrm{~L}_{2} \int_{0}^{\mathrm{t}} \mathrm{e}^{-2 \lambda(\mathrm{t}-\mathrm{s})} \mathrm{ds} \int_{0}^{\mathrm{t}} \mathrm{e}^{-2 \lambda(\mathrm{t}-\mathrm{s})} \mathbb{E}\|\mathrm{x}(\mathrm{s}-\delta(\mathrm{s}))\|^{2} \mathrm{ds} \\
\leq \frac{1}{2} \mathrm{M}^{2} \mathrm{~L}_{2} \lambda^{-1} \int_{0}^{\mathrm{t}} \mathrm{e}^{-2 \lambda(\mathrm{t}-\mathrm{s})}\left(\mathrm{M}^{*}+\mathbb{E}\|\phi\|_{\mathrm{c}}^{2}\right) \mathrm{e}^{\mathrm{a} \tau} \mathrm{e}^{-\mathrm{as}} \mathrm{ds} \\
\leq \frac{1}{2} \mathrm{M}^{2} \mathrm{~L}_{2} \lambda^{-1}(2 \lambda-\mathrm{a})^{-1}\left(\mathrm{M}^{*}+\mathbb{E}\|\phi\|_{\mathrm{c}}^{2}\right) \mathrm{e}^{\mathrm{a} \tau} \mathrm{e}^{-\mathrm{at}} \\
\leq \mathrm{M}_{5}^{*} \mathrm{e}^{-\mathrm{at}}
\end{gathered}
$$

By using lemma 2.2 we get that

$$
\begin{aligned}
\mathbb{E}\left\|p_{6}(\mathrm{t})\right\|^{2} & =\mathbb{E}\left\|\int_{0}^{\mathrm{t}} \mathrm{S}(\mathrm{t}-\mathrm{s}) \sigma(\mathrm{s}) \mathrm{dB}^{\mathrm{H}}(\mathrm{s})\right\|^{2} \\
& \leq \mathrm{M}^{2} \mathrm{cH}(2 \mathrm{H}-1) \mathrm{t}^{2 \mathrm{H}-1} \int_{0}^{\mathrm{t}} \mathrm{e}^{-2 \lambda(\mathrm{t}-\mathrm{s})}\|\sigma(\mathrm{s})\|_{\mathrm{L}_{\mathrm{Q}}^{0}}^{2} \mathrm{ds}
\end{aligned}
$$

From this inequality we can infer that

$\mathbb{E}\left\|\mathrm{p}_{6}(\mathrm{t})\right\|^{2} \leq \mathrm{M}^{2} \mathrm{cH}(2 \mathrm{H}-1) \mathrm{t}^{2 \mathrm{H}-1} \mathrm{e}^{-2 \lambda^{\prime} \mathrm{t}} \int_{0}^{\mathrm{t}} \mathrm{e}^{2 \gamma \mathrm{s}}\|\sigma(\mathrm{s})\|_{\mathcal{L}_{\mathrm{Q}}^{0}}^{2} \mathrm{ds}$

where $\lambda^{\prime}=\lambda \wedge \gamma$.Indeed, if $\lambda^{\prime}<\gamma$, then $\lambda^{\prime}=\lambda$ and we have

$$
\begin{aligned}
\mathbb{E}\left\|p_{6}(\mathrm{t})\right\|^{2} & \leq \mathrm{M}^{2} \mathrm{cH}(2 \mathrm{H}-1) \mathrm{t}^{2 \mathrm{H}-1} \mathrm{e}^{-2 \lambda \mathrm{t}} \int_{0}^{\infty} \mathrm{e}^{2 \lambda s}\|\sigma(\mathrm{s})\|_{\mathrm{L}_{\mathrm{Q}}^{0}}^{2} \mathrm{ds} \\
& \leq \mathrm{M}^{2} \mathrm{cH}(2 \mathrm{H}-1) \mathrm{t}^{2 \mathrm{H}-1} \mathrm{e}^{-2 \lambda^{\prime} \mathrm{t}} \int_{0}^{\infty} \mathrm{e}^{2 \gamma \mathrm{s}}\|\sigma(\mathrm{s})\|_{\mathcal{L}_{\mathrm{Q}}^{0}}^{2} \mathrm{ds}
\end{aligned}
$$

If $\gamma<\lambda$ then $\lambda^{\prime}=\gamma$ and we have 
$\mathbb{E}\left\|p_{6}(t)\right\|^{2} \leq M^{2} c H(2 H-1) t^{2 H-1} e^{-2 \lambda^{\prime} t} \int_{0}^{t} e^{2 \gamma s}\|\sigma(s)\|_{\mathcal{L}_{Q}^{0}}^{2} d s$

since $\sup _{\mathrm{t} \geq 0}\left(\mathrm{t}^{2 \mathrm{H}-1} \mathrm{e}^{-\lambda^{\prime} \mathrm{t}}\right)<\infty$, this together with (4.8) we have

$\mathbb{E}\left\|\mathrm{p}_{6}(\mathrm{t})\right\|^{2} \leq \mathrm{M}_{6}^{*} \mathrm{e}^{-\mathrm{at}}$

Combining (4.3)-(4.7), (4.9) we see that there exists $\overline{\mathrm{M}}^{*}>0$ and $\overline{\mathrm{a}}>0$ such that $\mathbb{E}\|(\Phi \mathrm{x})(\mathrm{t})\|^{2} \leq \overline{\mathrm{M}}^{*} \mathrm{e}^{-\mathrm{at}}, \mathrm{t} \geq 0$.

Hence we conclude that $\Phi(s) \subset s$.

Step 2 . We now show that $\Phi$ is a contraction mapping. For any $\mathrm{x}, \mathrm{y} \in \boldsymbol{s}$, we have

$$
\mathbb{E}\|(\Phi \mathrm{x})(\mathrm{t})-(\Phi \mathrm{y})(\mathrm{t})\|^{2} \leq 4 \sum_{\mathrm{i}=1}^{\mathrm{n}} \mathrm{I}_{\mathrm{i}}
$$

Since $x(t)=y(t)=\varphi(t), t \in(-\tau, 0]$, this implies that

$$
\mathbb{E}\|x(t-r(t))-y(t-r(t))\|^{2} \leq \sup _{t \geq 0} \mathbb{E}\|x(t)-y(t)\|^{2}
$$

Then we have by assumption(i) in $\left(\mathrm{H}_{3}\right)$

$$
\begin{aligned}
\mathrm{I}_{1} & =\mathbb{E}\|\mathrm{g}(\mathrm{t}, \mathrm{x}(\mathrm{t}-\mathrm{r}(\mathrm{t})))-\mathrm{g}(\mathrm{t}, \mathrm{y}(\mathrm{t}-\mathrm{r}(\mathrm{t})))\|^{2} \\
& \leq \mathrm{L}_{3}\left\|(-\mathrm{A})^{-\beta}\right\|^{2} \mathbb{E}\|\mathrm{x}(\mathrm{t}-\mathrm{r}(\mathrm{t}))-\mathrm{y}(\mathrm{t}-\mathrm{r}(\mathrm{t}))\|^{2} \\
& \leq \mathrm{L}_{3}\left\|(-\mathrm{A})^{-\beta}\right\|^{2} \sup _{\mathrm{t} \geq 0} \mathbb{E}\|\mathrm{x}(\mathrm{t})-\mathrm{y}(\mathrm{t})\|^{2} \\
\mathrm{I}_{2}= & \mathbb{E}\left\|\int_{0}^{\mathrm{t}} \mathrm{AS}(\mathrm{t}-\mathrm{s})[\mathrm{g}(\mathrm{s}, \mathrm{x}(\mathrm{s}-\mathrm{r}(\mathrm{s})))-\mathrm{g}(\mathrm{s}, \mathrm{y}(\mathrm{s}-\mathrm{r}(\mathrm{s})))] \mathrm{ds}\right\|^{2} \\
\leq & \mathrm{M}_{1-\beta}^{2} \mathrm{~L}_{3} \int_{0}^{\mathrm{t}}(\mathrm{t}-\mathrm{s})^{\beta-1} \mathrm{e}^{-\lambda(\mathrm{t}-\mathrm{s})} \mathrm{ds} \int_{0}^{\mathrm{t}}(\mathrm{t}-\mathrm{s})^{\beta-1} \mathrm{e}^{-\lambda(\mathrm{t}-\mathrm{s})} \mathbb{E}\|\mathrm{x}(\mathrm{s}-\mathrm{r}(\mathrm{s}))-\mathrm{y}(\mathrm{s}-\mathrm{r}(\mathrm{s}))\|^{2} \mathrm{ds} \\
\leq & M_{1-\beta}^{2} \mathrm{~L}_{3} \frac{\Gamma(\beta)}{\lambda^{\beta}} \int_{0}^{\mathrm{t}}(\mathrm{t}-\mathrm{s})^{\beta-1} \mathrm{e}^{-\lambda(\mathrm{t}-\mathrm{s})} \mathbb{E}\|\mathrm{x}(\mathrm{s}-\mathrm{r}(\mathrm{s}))-\mathrm{y}(\mathrm{s}-\mathrm{r}(\mathrm{s}))\|^{2} \mathrm{ds} \\
\leq & M_{1-\beta}^{2} \mathrm{~L}_{3} \frac{\Gamma^{2}(\beta)}{\lambda^{2 \beta}} \sup _{\mathrm{t} \geq 0} \mathbb{E}\|\mathrm{x}(\mathrm{t})-\mathrm{y}(\mathrm{t})\|^{2}
\end{aligned}
$$

By assumption (i) in $\left(\mathrm{H}_{2}\right)$

$$
\begin{aligned}
I_{3} & =\mathbb{E}\left\|\int_{0}^{t} S(t-s)[f(s, x(s-\rho(s)))-f(s, y(s-\rho(s)))] d s\right\| \\
& \leq M^{2} L_{1} \int_{0}^{t} e^{-\lambda(t-s)} d s \int_{0}^{t} e^{-\lambda(t-s)} \mathbb{E}\|x(s-\rho(s))-y(s-\rho(s))\|^{2} d s \\
& \leq M^{2} L_{1} \lambda^{-1} \int_{0}^{t} e^{-\lambda(t-s)} \mathbb{E}\|x(s-\rho(s))-y(s-\rho(s))\|^{2} d s \\
& \leq M^{2} L_{1} \lambda^{-2} \sup _{t \geq 0} \mathbb{E}\|x(t)-y(t)\|^{2}
\end{aligned}
$$

By assumption(iii) in $\left(\mathrm{H}_{2}\right)$, we have

$$
\begin{aligned}
\mathrm{I}_{4} & =\mathbb{E}\left\|\int_{0}^{\mathrm{t}} \mathrm{S}(\mathrm{t}-\mathrm{s})[\mathrm{h}(\mathrm{s}, \mathrm{x}(\mathrm{s}-\delta(\mathrm{s})))-\mathrm{h}(\mathrm{s}, \mathrm{x}(\mathrm{s}-\delta(\mathrm{s})))] \mathrm{dw}(\mathrm{s})\right\| \\
& \leq \mathrm{M}^{2} \mathrm{~L}_{2} \int_{0}^{\mathrm{t}} \mathrm{e}^{-2 \lambda(\mathrm{t}-\mathrm{s})} \mathrm{ds} \int_{0}^{\mathrm{t}} \mathrm{e}^{-2 \lambda(\mathrm{t}-\mathrm{s})} \mathbb{E}\|\mathrm{x}(\mathrm{s}-\delta(\mathrm{s}))-\mathrm{y}(\mathrm{s}-\delta(\mathrm{s}))\|^{2} \mathrm{ds} \\
& \leq \mathrm{M}^{2} \mathrm{~L}_{2}(2 \lambda)^{-1} \int_{0}^{\mathrm{t}} \mathrm{e}^{-2 \lambda(\mathrm{t}-\mathrm{s})} \mathbb{E}\|\mathrm{x}(\mathrm{s}-\delta(\mathrm{s}))-\mathrm{y}(\mathrm{s}-\delta(\mathrm{s}))\|^{2} \mathrm{ds} \\
& \leq \mathrm{M}^{2} \mathrm{~L}_{2}(2 \lambda)^{-2} \sup _{\mathrm{t} \geq 0} \mathbb{E}\|\mathrm{x}(\mathrm{t})-\mathrm{y}(\mathrm{t})\|^{2}
\end{aligned}
$$


$\operatorname{ThusE}\|(\Phi x)(t)-(\Phi y)(t)\|^{2}$

$$
\leq 4\left(\mathrm{~L}_{3}\left\|(-\mathrm{A})^{-\beta}\right\|^{2}+\mathrm{M}_{1-\beta}^{2} \mathrm{~L}_{3} \frac{\Gamma^{2}(\beta)}{\lambda^{2 \beta}}+\mathrm{M}^{2} \mathrm{~L}_{1} \lambda^{-2}+\mathrm{M}^{2} \mathrm{~L}_{2}(2 \lambda)^{-2}\right) \sup _{\mathrm{t} \geq 0} \mathbb{E}\|\mathrm{x}(\mathrm{t})-\mathrm{y}(\mathrm{t})\|^{2}
$$

By the condition (4.1), we claim that $\Phi$ is a contractive. So, applying the Banach fixed point principle, the proof is complete.

Now we consider the case of infinite delays. We assume that $t-r(t) \rightarrow \infty, t-\rho(t) \rightarrow \infty, t-\delta(t) \rightarrow \infty$ as $t \rightarrow \infty$.

Theorem 4.2:[Infinite delays]Under the conditions of theorem 4.1, the mild solution to (1.1) exists uniquely and converges to zero in mean square i.e., $\lim _{\mathrm{t} \rightarrow \infty} \mathbb{E}\|\mathrm{x}(\mathrm{t})\|^{2}=0$

Proof: Denote by $s^{\prime}$ the space of all stochastic processes $x(t, \omega):(-\infty, \infty) \times \Omega \rightarrow X$ satisfying

$x(t)=\phi(t), t \in(-\infty, 0]$ and

$\lim _{t \rightarrow \infty} \mathbb{E}\|x(t)\|^{2}=0$

We define the operator $\Psi$ on $s^{\prime}$ by $(\Psi x)(t)=\phi(t), t \in(-\infty, 0]$ and

$$
\begin{aligned}
(\Psi x)(t) & =S(t)(\phi(0)+g(0, \phi(-r(0))))-g(t, x(t-r(t))) \\
& -\int_{0}^{t} A S(t-s) g(s, x(s-r(s))) d s+\int_{0}^{t} S(t-s) f(s, x(s-\rho(s))) d s \\
& +\int_{0}^{t} S(t-s) h(s, x(s-\delta(s))) d w(s)+\int_{0}^{t} S(t-s) \sigma(s) d B^{H}(s)
\end{aligned}
$$

Since $(\Psi x)(t)=(\Phi x)(t)$ on $[0, \infty)$, this implies that $\Psi$ is contractive. Hence it remains to check $\Phi\left(s^{\prime}\right) \subset s^{\prime}$. In order to obtain this claim, we need to show that $\lim _{\mathrm{t} \rightarrow \infty} \mathbb{E}\|\Psi \mathrm{x}(\mathrm{t})\|^{2}=0$ for all $x \in s^{\prime}$.

By the definition of $s^{\prime}$, assumption $\left(\mathrm{H}_{7}\right)$ and the fact $\mathrm{t}-\mathrm{r}(\mathrm{t}) \rightarrow \infty, \mathrm{t} \rightarrow \infty$. We get

$$
\lim \mathbb{E}_{t \rightarrow \infty}\left\|P_{1}(t)\right\|^{2}=\lim _{t \rightarrow \infty} \mathbb{E}\left\|P_{2}(t)\right\|^{2}=\lim _{t \rightarrow \infty} \mathbb{E}\left\|P_{6}(t)\right\|=0
$$

We further have $\mathbb{E}\left\|p_{3}(t)\right\|^{2} \leq \mathbb{E}\left\|\int_{0}^{t} A S(t-s) g(s, x(s-r(s))) d s\right\|^{2}$

$$
\begin{aligned}
& \leq M_{1-\beta}^{2} L_{3} \int_{0}^{t}(t-s)^{\beta-1} e^{-\lambda(t-s)} d s \int_{0}^{t}(t-s)^{\beta-1} e^{-\lambda(t-s)} \mathbb{E}\|x(s-r(s))\|^{2} d s \\
& \leq M_{1-\beta}^{2} L_{3} \Gamma(\beta) \lambda^{-\beta} \int_{0}^{t}(t-s)^{\beta-1} e^{-\lambda(t-s)} E\|x(s-r(s))\|^{2} d s
\end{aligned}
$$

For any $\mathrm{x} \in \mathrm{s}^{\prime}$ and $\varepsilon>0$ it follows from (4.10) that there exists $\mathrm{s}_{1}>0$ such that $\mathbb{E}\|\mathrm{x}(\mathrm{s}-\mathrm{r}(\mathrm{s}))\|^{2}<\epsilon$ for all $\mathrm{s} \geq \mathrm{s}_{1}$. Thus we obtain

$\mathbb{E}\left\|p_{3}(t)\right\|^{2} \leq M_{1-\beta}^{2} L_{3} \Gamma(\beta) \lambda^{-\beta} \int_{0}^{s_{1}}(t-s)^{\beta-1} e^{-\lambda(t-s)} \mathbb{E}\|x(s-r(s))\|^{2} d s+M_{1-\beta}^{2} L_{3} \Gamma^{2}(\beta) \lambda^{-2 \beta} \epsilon$

which proves that

$$
\begin{gathered}
\lim _{\mathrm{t} \rightarrow \infty} \mathbb{E}\left\|\mathrm{P}_{3}(\mathrm{t})\right\|^{2} \leq \mathrm{M}_{1-\beta}^{2} \mathrm{~L}_{3} \Gamma^{2}(\beta) \lambda^{-2 \beta}, \forall \epsilon>0 \text { and hence, } \lim _{\mathrm{t} \rightarrow \infty} \mathbb{E}\left\|\mathrm{P}_{3}(\mathrm{t})\right\|^{2}=0 \\
\mathbb{E}\left\|\mathrm{p}_{4}(\mathrm{t})\right\|^{2}=\mathbb{E}\left\|\int_{0}^{\mathrm{t}} \mathrm{S}(\mathrm{t}-\mathrm{s}) \mathrm{f}(\mathrm{s}, \mathrm{x}(\mathrm{s}-\rho(\mathrm{s}))) \mathrm{ds}\right\|^{2} \\
\leq \mathrm{M}^{2} \mathrm{~L}_{1} \int_{0}^{\mathrm{t}} \mathrm{e}^{-\lambda(\mathrm{t}-\mathrm{s})} \mathrm{ds} \int_{0}^{\mathrm{t}} \mathrm{e}^{-\lambda(\mathrm{t}-\mathrm{s})} \mathbb{E}\|\mathrm{x}(\mathrm{s}-\rho(\mathrm{s}))\|^{2} \mathrm{ds} \\
\leq \mathrm{M}^{2} \mathrm{~L}_{1} \lambda^{-1} \int_{0}^{\mathrm{t}} \mathrm{e}^{-\lambda(\mathrm{t}-\mathrm{s})} \mathbb{E}\|\mathrm{x}(\mathrm{s}-\rho(\mathrm{s}))\|^{2} \mathrm{ds}
\end{gathered}
$$


For any $\mathrm{x} \in \mathrm{s}^{\prime}$ and $\varepsilon>0$ there exists a $\mathrm{s}_{1}>0$ such that $\mathbb{E}\|\mathrm{x}(\mathrm{s}-\mathrm{r}(\mathrm{s}))\|^{2}<\epsilon$ for all $\mathrm{s} \geq \mathrm{s}_{1}$.

Thus we obtain $\mathbb{E}\left\|\mathrm{p}_{4}(\mathrm{t})\right\|^{2} \leq \mathrm{M}^{2} \mathrm{~L}_{1} \lambda^{-1} \int_{0}^{\mathrm{s}_{1}} \mathrm{e}^{-\lambda(\mathrm{t}-\mathrm{s})} \mathbb{E}\|\mathrm{x}(\mathrm{s}-\rho(\mathrm{s}))\|^{2} \mathrm{ds}+\mathrm{M}^{2} \mathrm{~L}_{1} \lambda^{-2} \epsilon$

which proves that $\lim _{\mathrm{t} \rightarrow \infty} \mathrm{E}\left\|\mathrm{P}_{4}(\mathrm{t})\right\|^{2} \leq \mathrm{M}^{2} \mathrm{~L}_{1} \lambda^{-2} \epsilon, \forall \epsilon>0$ and hence, $\mathrm{im}_{\mathrm{t} \rightarrow \infty} \mathrm{E}\left\|\mathrm{P}_{4}(\mathrm{t})\right\|^{2}=0$

$$
\begin{aligned}
\mathbb{E}\left\|p_{5}(t)\right\|^{2} & =\mathbb{E}\left\|\int_{0}^{t} S(t-s) h(s, x(s-\delta(s))) d w(s)\right\|^{2} \\
& \leq M^{2} L_{2} \int_{0}^{t} e^{-2 \lambda(t-s)} \mathbb{E}\|x(s-\delta(s))\|^{2} d s
\end{aligned}
$$

For any $\mathrm{x} \in \mathrm{s}^{\prime}$ and $\varepsilon>0$ there exists as $\mathrm{s}_{1}>0$ such that $\mathbb{E}\|\mathrm{x}(\mathrm{s}-\mathrm{r}(\mathrm{s}))\|^{2}<\epsilon$ for all $\mathrm{s} \geq \mathrm{s}_{1}$.

Thus we obtain $\mathbb{E}\left\|\mathrm{p}_{5}(\mathrm{t})\right\|^{2} \leq \mathrm{M}^{2} \mathrm{~L}_{2} \int_{0}^{\mathrm{s}_{1}} \mathrm{e}^{-2 \lambda(\mathrm{t}-\mathrm{s})} \mathbb{E}\|\mathrm{x}(\mathrm{s}-\delta(\mathrm{s}))\|^{2} \mathrm{ds}+\mathrm{M}^{2} \mathrm{~L}_{2}(2 \lambda)^{-1} \epsilon$

which proves that $\lim _{\mathrm{t} \rightarrow \infty} \mathbb{E}\left\|\mathrm{P}_{5}(\mathrm{t})\right\|^{2} \leq \mathrm{M}^{2} \mathrm{~L}_{2}(2 \lambda)^{-1} \epsilon, \forall \epsilon>0$ and hence, $\lim _{\mathrm{t} \rightarrow \infty} \mathbb{E}\left\|\mathrm{P}_{5}(\mathrm{t})\right\|^{2}=0$

Once again, by applying the Banach fixed point principle, we complete the proof of the theorem.

To illustrate the obtained theory, let us end this section with an example.

\section{Example}

Consider the following neutral stochastic partial differential equations with delays driven by a fBm in the following form

$$
\frac{\partial}{\partial \mathrm{t}}\left[\mathrm{z}(\mathrm{t}, \xi)+\alpha_{1} \mathrm{G}(\mathrm{t}, \mathrm{z}(\mathrm{t}-\tau, \xi))\right]=\frac{\partial^{2}}{\partial \xi^{2}} \mathrm{z}(\mathrm{t}, \xi)+\alpha_{2} \mathrm{~F}(\mathrm{t}, \mathrm{z}(\mathrm{t}-\tau, \xi)) \partial \mathrm{t}+\alpha_{3} \varphi(\mathrm{t}, \mathrm{z}(\mathrm{t}-\tau, \xi)) \mathrm{dw}(\mathrm{t})+\Theta(\mathrm{t}) \mathrm{dB} \mathrm{B}_{\mathrm{Q}}^{\mathrm{H}}(\mathrm{t})
$$

$\mathrm{z}(\mathrm{t}, 0)=\mathrm{z}(\mathrm{t}, \pi)=0$

$\mathrm{z}(\mathrm{t}, \xi)=\phi(\mathrm{t}, \xi), \mathrm{t} \in(-\infty, 0], 0 \leq \xi \leq \pi$

where $\alpha_{1}, \alpha_{2}, \alpha_{3}>0$ are constants.

Let $\mathrm{X}=\mathrm{L}^{2}(0, \pi)$ with the norm $\|\cdot\|$ and inner product $\langle\cdot ;\rangle$.Define $\mathrm{A}: \mathrm{X} \rightarrow \mathrm{X}$ by $\mathrm{Ax}=\mathrm{x}$ " with domain

$D(A)=\left\{x \in X: X, x^{\prime}\right.$ are absolutely continuous $\left.x^{\prime \prime} \in X, X(0)=x(\pi)=0\right\}$. Then Eqn. (5.1) can be written in the form of equation (1.1) with the co-efficient $\mathrm{g}(\mathrm{t}, \mathrm{x})=\alpha_{1} \mathrm{G}(\mathrm{t}, \mathrm{z}(\mathrm{t}-\tau, \xi)), \mathrm{f}(\mathrm{t}, \mathrm{x})=\alpha_{2} \mathrm{~F}(\mathrm{t}, \mathrm{z}(\mathrm{t}-\tau, \xi))$ and

$\mathrm{h}(\mathrm{t}, \mathrm{x})=\alpha_{3} \varphi(\mathrm{t}, \mathrm{z}(\mathrm{t}-\tau, \xi)), \sigma(\mathrm{t})=\Theta(\mathrm{t})$.

For the operator A, it is known from Pazy [18] that the following properties hold.

$A x=\sum_{n=1}^{\infty} A^{2}\left\langle x, e_{n}\right\rangle e_{n}, x \in D(A)$, where $e_{n}(t)=\sqrt{\frac{2}{\pi}} \sin (n t), n=1,2,3, \ldots$ is the orthogonal set of eigenvectors.

$* A$ is the infinitesimal generator of an analytic semigroup $(S(t))_{t \geq 0}$ in $X$.

$S(t) x=\sum_{n=1}^{\infty} e^{-n^{2} t}\left\langle x, e_{n}\right\rangle e_{n}$, For all $x \in X$ and for every $t>0$.

Furthermore $\|S(\mathrm{t})\| \leq \mathrm{e}^{-\pi^{2} \mathrm{t}}, \mathrm{t} \geq 0$.

The bounded linear operator $(-\mathrm{A})^{3 / 4}$ is well defined and is given by

$$
(-A)^{3 / 4} x=\sum_{n=1}^{\infty}(n)^{3 / 2}\left\langle x, e_{n}\right\rangle e_{n}
$$

with domain $\mathrm{D}\left((-\mathrm{A})^{3 / 4}\right)=\left\{\mathrm{x} \in \mathrm{X}: \sum_{\mathrm{n}=1}^{\infty} \mathrm{e}^{-\mathrm{n}^{2} \mathrm{t}}\left\langle\mathrm{x}, \mathrm{e}_{\mathrm{n}}\right\rangle \mathrm{e}_{\mathrm{n}} \in \mathrm{X}\right\}$. Furtheremore $\left\|(-\mathrm{A})^{3 / 4}\right\|=1$ and $\left\|(-\mathrm{A})^{3 / 4}\right\| \leq$ $\frac{1}{\Gamma(3 / 4)} \int_{0}^{\infty} \mathrm{t}^{-(1 / 4)}\|\mathrm{S}(\mathrm{t})\| \mathrm{dt}<\frac{1}{\pi^{3 / 2}}$. Thus $\left(\mathrm{H}_{1}\right)$ holds with $\mathrm{M}=1, \lambda=\pi^{2},\left(\mathrm{H}_{2}\right)$ holds with $\mathrm{L}_{1}=\alpha_{2}{ }^{2},\left(\mathrm{H}_{3}\right)$ and $\left(\mathrm{H}_{4}\right)$ holds with $\beta=3 / 4, \mathrm{~L}_{2}=\left\|(-\mathrm{A})^{3 / 4}\right\|^{2} \alpha_{1}{ }^{2}=\alpha_{1}{ }^{2}$ and $\left(\mathrm{H}_{6}\right)$ holds with $\gamma=\frac{1}{2}$.Consequently we conclude by theorem 4.2 , that the stochastic partial equation (5.1) has a unique mild solution and that this solution converges to zero in mean square if the parameters $\alpha_{1}, \alpha_{2}, \alpha_{3}$ satisfy the following conditions.

$$
\frac{\alpha_{1}^{2}}{\pi^{3}}+\frac{\mathrm{M}_{1}^{2}}{4} \Gamma^{2}\left(\frac{3}{4}\right) \frac{\alpha_{1}^{2}}{\pi^{3}}+\frac{\alpha_{2}^{2}}{\pi^{4}}+\frac{\alpha_{3}^{2}}{\pi^{4}}<\frac{1}{4}
$$




\section{Conclusion}

In this paper we derived existence, conditions ensuring the exponential decay to zero in mean square of a neutral stochastic delay differential equations driven by $\mathrm{fBm}$. In addition we also established the case of infinite delays which has not yet been discussed in the context of neutral stochastic delay differential equations driven by $\mathrm{fBm}$.

\section{References}

[1] L. Coutin, Z. Qian, Stochastic differential equations for fractional Brownian motions. ComptesRendus de I'Académie des Sciences-Series IMathematics 331 (2000) 75-80. http://dx.doi.org/10.1016/S0764-4442(00)01594-9.

[2] Y. Hu, D. Nualart, Differential equations driven by H*older continuous functions of order greater than $1 / 2$, Stochastic analysis and applications 2 (2007) 399-413. http://dx.doi.org/10.1007/978-3-540-70847-6 17.

[3] D. Nualart, Y. Ouknine, Regularization of differential equations by fractional noise, Stochastic Process and their Applications 102 (2002) 103 116. http://dx.doi.org/10.1016/S0304-4149(02)00155-2.

[4] D. Nualart, A. R`a,scanu, Differential equations driven by fractional Brownian motion, CollectaneaMathematica 53 (2002) 55-81.

[5] D. Nualart, B. Saussereau, Malliavin calculus for stochastic differential equations driven by a fractional Brownian motion, Stochastic Processes and their Applications119 (2009) 391-409. http://dx.doi.org/10.1016/j.spa.2008.02.016.

[6] T. Caraballo, J. Real, T. Taniguchi, The exponential stability of neutral stochastic delay partial differential equations, Discrete and Continuous Dynamical Systems 18 (2007) 295-313. http://dx.doi.org/10.3934/dcds.2007.18.295.

[7] W. Dai, C. C. Heyde, Ito's formula with respect to fractional Brownian motion and its application, Journal of Applied Mathematics and Stochastic Analysis 9 (1996) 439-448. http://dx.doi.org/10.1155/S104895339600038X.

[8] D. Feyel, A. de la Pradelle, on fractional Brownian processes, Potential Analysis $10 \quad(1999) 273-288$. http://dx.doi.org/10.1023/A:1008630211913.

[9] E. Hernandez, D. N. Keck, M. A. McKibben, On a class of measure-dependent stochastic evolution equations driven by fBm, Journal of Applied Mathematics and Stochastic Analysis 2007 (2007) 1-26. http://dx.doi.org/10.1155/2007/69747.

[10] P. H. Bezandry, Existence of almost periodic solutions for semilinear stochastic evolution equations driven by fractional Brownian motion, Electronic Journal of Differential Equations 2012 (2012) 1-21.

[11] T. E. Duncan, B. Maslowski, B. Pasik-Duncan, Fractional Brownian motion and stochastic equations in Hilbert spaces, Stochastics and Dynamics 2 (2002) 225-250. http://dx.doi.org/10.1142/S0219493702000340.

[12] B. Maslowski, D. Nualart, Evolution equations driven by a fractional Brownian motion, Journal of Functional Analysis 2 (2003) $277-305$. http://dx.doi.org/10.1016/S0022-1236(02)00065-4.

[13] T. Caraballo, M. J. Garrido-Atienza, T. Taniguchi, The existence and exponential behavior of solutions to stochastic delay evolution equations with a fractional Brownian motion, Nonlinear Analysis: Theory, Methods and Applications 74 (2011) 3671-3684. http://dx.doi.org/10.1016/j.na.2011.02.047.

[14] B. Boufoussi, S. Hajji, Neutral stochastic functional differential equations driven by a fractional Brownian motion in a Hilbert space, Statistics \& Probability Letters 82 (2012)1549-1558. http://dx.doi.org/10.1016/j.spl.2012.04.013.

[15] T. E. Duncan, B. Maslowski, B. Pasik-Duncan, Stochastic equations in Hilbert space with a multiplicative fractional Gaussian noise, Stochastic process and their applications 115(8) (2005) 1357-1383.

[16] Nguyen-Tien-Dung, Neutral stochastic differential equations driven by a fractional Brownian motion with impulsive effects and varying time delays, Journal of the Korean Statistical society 43 (2014) 599-608. http://dx.doi.org/10.1016/j.jkss.2014.02.003.

[17] T. A. Burton. Stability by fixed point theory for functional differential equations, Mineola, NY, Dover Publications, Inc. (2006).

[18] A. Pazy, Semigroups of Linear Operators and Applications to Partial Differential Equations, Applied Mathematical Sciences, vol. 44, Springer-Verlag, New York. (1983). http://dx.doi.org/10.1007/978-1-4612-5561-1.

[19] G. Da Prato, J. Zabczyk, Stochastic equations in infinite dimensions, Cambridge University Press, Cambridge, (1992). http://dx.doi.org/10.1017/CBO9780511666223. 